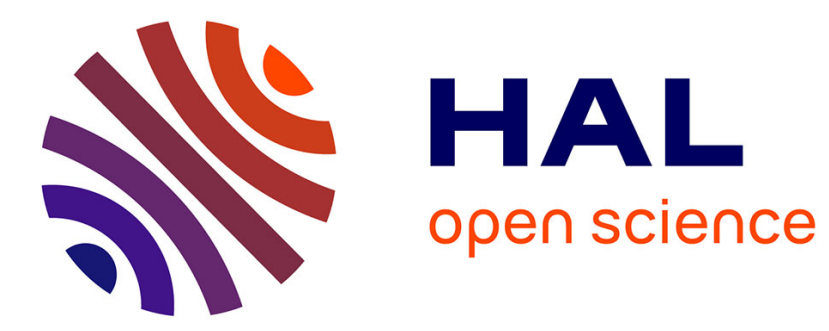

\title{
Measurement of Dipeptidylpeptidase Activity in vitro and in vivo
}

Rosa Barreira da Silva, Molly Ingersoll, Matthew Albert

\section{To cite this version:}

Rosa Barreira da Silva, Molly Ingersoll, Matthew Albert. Measurement of Dipeptidylpeptidase Activity in vitro and in vivo. Bio-protocol , 2017, 7 (6), pp.e2184. 10.21769/BioProtoc.2184 . pasteur03331047

\section{HAL Id: pasteur-03331047 \\ https://hal-pasteur.archives-ouvertes.fr/pasteur-03331047}

Submitted on 1 Sep 2021

HAL is a multi-disciplinary open access archive for the deposit and dissemination of scientific research documents, whether they are published or not. The documents may come from teaching and research institutions in France or abroad, or from public or private research centers.
L'archive ouverte pluridisciplinaire HAL, est destinée au dépôt et à la diffusion de documents scientifiques de niveau recherche, publiés ou non, émanant des établissements d'enseignement et de recherche français ou étrangers, des laboratoires publics ou privés. 


\title{
Measurement of Dipeptidylpeptidase Activity in vitro and in vivo
}

Rosa Barreira da Silva ${ }^{1}$, Molly A. Ingersoll ${ }^{2,3}$ and Matthew L. Albert ${ }^{1,2,3, *}$

\author{
${ }^{1}$ Cancer Immunology, Genentech, South San Francisco, CA, USA; ${ }^{2}$ Laboratory of Dendritic Cell \\ Immunobiology, Institut Pasteur, Paris, France; ${ }^{3}$ INSERM U1223, Paris, France
}

*For correspondence: albertm7@gene.com

\begin{abstract}
[Abstract] Dipeptidylpeptidases (DPPs) are serine proteases, which cleave small proteins and peptides possessing a proline or an alanine in the second position of their $\mathrm{N}$-terminus. Among the members of this family, dipeptidylpeptidase 4 (DPP4) is constitutively expressed in the extracellular space. DPP4 is found at the surface of many hematopoietic and non-hematopoietic cells and is also present in many biological fluids in a bioactive soluble form. DPP4 expression is modulated by inflammation, and measurements of its activity have been used as biomarker for disease. Here, we describe a method to evaluate the enzymatic activity of DPP4 in vitro and in vivo.
\end{abstract}

Keywords: Dipeptidylpeptidase 4, Enzyme, Tumor, Plasma, DPP4 inhibitor

[Background] The magnitude of the enzymatic activity of DPPs can be an indicator of inflammation; and an important pharmacodynamics parameter for usage of DPP4 inhibitors. Here, we report details for the usage of a commercially available kit to evaluate DPP-enzymatic activity in vitro; and provide details on the generation of biological samples tested. Furthermore, we describe how this method can be used for evaluation of DPP activity in vivo.

\section{Materials and Reagents}

1. 96 well plates, $U$ bottom (Fisher Scientific, catalog number: 12-565-500)

2. Luminometer 96 well plates (Promega, catalog number: Z3291)

3. $2 \mathrm{ml}$ Eppendorf tube (Fisher Scientific, catalog number: 05-402-7)

4. $1 \mathrm{ml}$ syringe (BD, catalog number: 309659$)$

5. $20 \mathrm{G}(0.9 \times 25 \mathrm{~mm})$ needle (BD, catalog number: 305175$)$

6. Luciferase-expressing transgenic mouse such as the FVB-Tg(CAG-luc,-GFP)L2G85Chco/J mouse (THE JACKSON LABORATORIES, catalog number: 008450)

7. Biologic samples such as mouse plasma/serum or organ homogenates/supernatants (see Notes)

8. DPPIV-Glo ${ }^{\mathrm{TM}}$ Protease Assay (Promega, catalog number: G8351)

9. $1 \times$ PBS (Thermo Fisher Scientific, Gibco ${ }^{T M}$ catalog number: 14190144)

10. Recombinant human DPP4 (R\&D Systems, catalog number: 1180-SE-010)

11. Prionex (Sigma-Aldrich, catalog number: G0411)

12. DPP4 inhibitor (such as K579, Sigma-Aldrich, catalog number: D3572) 
13. Ethanol

\section{Equipment}

1. Luminometer, with plate reader (such as Promega, model: GloMax ${ }^{\circledR} 96$ Microplate Luminometer)

2. Multichannel pipet

3. XENOGEN (PerkinElmer, model: IVIS system)

4. Precision scale (such as Veritas Analytical Balance, AFFORDABLE SCALES AND BALANCES, model: M124A)

5. Sterile stainless steel bead $5 \mathrm{~mm}$ (QIAGEN, catalog number: 69989)

6. TissueLyser II (QIAGEN, catalog number: 85300)

7. Tabletop microcentrifuge (such as Eppendorf, model: $5417 \mathrm{R}$ )

\section{Procedure}

A. Measuring DPP4 activity in vitro

1. The protocol to measure DPP4 enzymatic activity was performed as described by Promega (provider of the DPP4 enzymatic activity kit used in our assays). Briefly, this assay provides a DPP4-sensitive substrate (Gly-Pro-aminoluciferin) that produces a luminescent signal upon reaction with dipeptidylpeptidases (DPP) and luciferase. DPP-mediated truncation of the GlyPro dipeptide is necessary for this reaction, and therefore the luminescent signal is indicative of DPP enzymatic activity. Important considerations concern the preparation and dilutions of biologic samples used in these assays:

a. Mouse serum and plasma samples should be used at 1 to 20 dilution in Prionex solution.

b. Mouse tumors homogenates should be used at 1 to 2 and 1 to 10 dilution in Prionex solution.

Note: Please refer to the notes at the end of the protocol for instructions on how to obtain tumor homogenates.

c. Peritoneal washes (collected with $1 \mathrm{ml}$ of sterile cold PBS) should be used at 1 to 2 dilution in Prionex solution.

d. A standard curve should be performed using dilutions of recombinant human DPP4 in Prionex solution. Start at $50 \mathrm{ng} / \mathrm{ml}$ and perform 2 fold dilutions until $0.01 \mathrm{ng} / \mathrm{ml}$.

e. Leave 2 wells with Prionex solution only (blank wells) in order to determine the background values of the assay.

Note: We recommend the dilutions to be done in a 96-well U-bottom plate, before transferring them into the luminometer plate. Prepare the samples in a final volume of at least $60 \mu \mathrm{l}$ in order to correctly pipet $50 \mu$ of each sample onto the luminometer plate. Transfer of samples should be done with a multichannel pipet, avoiding the generation of bubbles. When all the samples have been transferred, add $50 \mu \mathrm{l}$ of reaction buffer (provided in the kit) to each well, using a 
multichannel pipet. Pipetting up and down is not necessary. Before reading the results in the luminometer, incubate plate at room temperature for $15 \mathrm{~min}$, exposed to room light (do not cover the plate with light blocking foil).

2. Preparing tumor lysates for analysis of DPP4 activity

a. Collect tumors using sterile dissection tools and place them in cold, sterile $1 \times$ PBS.

b. Carefully dry the tumor with a paper towel and weigh it in a precision scale (such as Veritas Analytical Balance, M124A).

c. Place tumors in a $2 \mathrm{ml}$ Eppendorf tube. Note: Tumor can be frozen at $-20^{\circ} \mathrm{C}$ after this step.

d. To each tube, add a sterile stainless steel bead ( $5 \mathrm{~mm}$ ) and $250 \mu \mathrm{l}$ of cold sterile $1 \times$ PBS.

e. Tumor lysates are generated by high speed shaking, using the TissueLyser II. Speed used is $25 \mathrm{~Hz}$ for $2 \mathrm{~min}$. Repeat this step 2 times. The machine should be placed in a cold room. From this moment on, keep the samples on ice at all times.

f. Centrifuge tubes in an Eppendorf centrifuge at $500 \times \mathrm{g}$ for $10 \mathrm{~min}$, at $4{ }^{\circ} \mathrm{C}$.

g. Collect supernatants containing the tumor lysates into new, sterile tubes.

Note: It is advisable to make at least 2 aliquots from each supernatant, to avoid multiple freeze/thaw cycles in the future analysis.

h. Freeze supernatants at $-20^{\circ} \mathrm{C}$ or use them for evaluation of DPP4 activity.

3. Collecting peritoneal washes for analysis of DPP4 activity

a. Sacrifice mice according to the regulations instituted by your working facility. If possible, we recommend the mice to be sacrificed in a $\mathrm{CO}_{2}$ chamber, as cervical dislocation might result in internal organ damage and bleeding into the peritoneal cavity.

b. Disinfect the skin of the mouse with ethanol and cut it. Expose the entire peritoneal area, without damaging the peritoneal membrane.

c. Inject $1 \mathrm{ml}$ of cold $1 \times$ PBS, into the peritoneal cavity using a $1 \mathrm{ml}$ syringe with a $20 \mathrm{G}(0.9 \mathrm{x}$ $25 \mathrm{~mm}$ ) needle. We recommend the injection to be performed through the abdominal fat pads in order to reduce the probability of damaging organs.

d. Collect the peritoneal wash: hold up the peritoneal membrane over the intestine (use blunt tweezers) and gently aspirate the liquid with the same syringe used to inject the PBS.

e. Place each peritoneal wash in a $2 \mathrm{ml}$ Eppendorf tube.

f. Centrifuge tubes in a tabletop microcentrifuge (such as centrifuge $5417 \mathrm{R}$, Eppendorf) at $500 \times g$ for 5 min at $4{ }^{\circ} \mathrm{C}$. This step allows elimination of peritoneal cells collected with the washes.

g. Collect supernatants containing the peritoneal washes proteins into new, Eppendorf tubes. Note: It is advisable to make at least 2 aliquots from each supernatant, to avoid multiple freeze/thaw cycles in the future analysis.

h. Freeze peritoneal washes at $-20^{\circ} \mathrm{C}$ or use them for evaluation of DPP4 activity. 
B. Measuring DPP activity in vivo

The luciferin/luciferase system used to measure DPP activity in vitro can also be used to detect DPP activity in vivo, as follows:

1. Inject $50 \mu \mathrm{l}$ of $10 \mathrm{nM}$ Gly-Pro-aminoluciferin (provided by the Promega kit) into a luciferase expressing mouse, such as the FVB-Tg(CAG-luc) mice. Injection can be intravenous or intraperitoneal. Intravenous injection results in fast systemic delivery (within $5 \mathrm{~min}$, see Figure 1), while intraperitoneal injection results in luminescence detected in the peritoneal cavity area (Barreira da Silva et al., 2015).

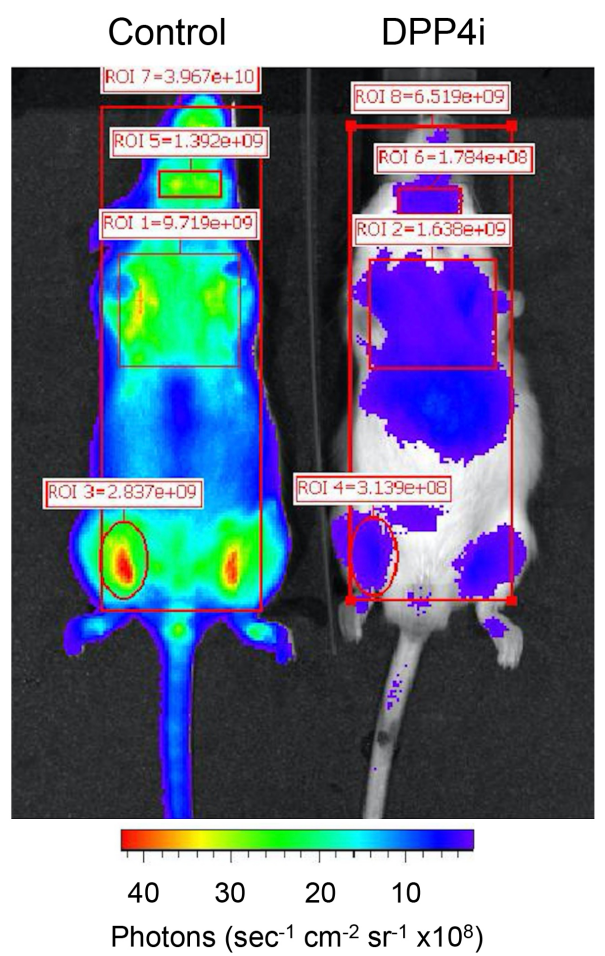

Figure 1. Example of luminescence image of FVB CAG-luciferase transgenic mice treated with control chow or chow containing DPP4 inhibitor (DPP4i). Mice were fed overnight with respective diets. DPP4i chow was sitagliptin 1.1\%. Injection of Gly-Proaminoluciferin was done intravenously and images were acquired 3 minutes after injection. Exposition time was $1 \mathrm{sec}$. Regions of interest (ROI) were selected for quantification of radiance, calculated in photons per second per $\mathrm{cm}^{2}$ (of area selected), per steradian ( $\mathrm{sec}^{-1} \mathrm{~cm}^{-}$ $\left.{ }^{2} \mathrm{sr}^{-1}\right)$.

2. Place the mouse in a XENOGEN (IVIS System) and image luminescence 3-5 min after injection. The mouse should be anesthetized during the entire procedure, using an isoflurane-based anesthesia system, as specified for each research institution. For image acquisition, test different exposure times (start with $1 \mathrm{sec}$, up to $1 \mathrm{~min}$ ). 


\section{Data analysis}

1. It is important to keep in mind that this assay detects the activity DPP4, but other members of the DPP family may also truncate Gly-Pro-aminoluciferin. In mouse serum or plasma samples, at least $95 \%$ of the measured DPP activity corresponds to DPP4, as evaluated by comparing samples from wildtype and dpp $4^{-1-}$ mice. Whenever evaluating the specific contribution of DPP4 in other biologic samples (e.g., tumor lysates), we advise assaying the sample in duplicate and adding a DPP4 inhibitor (such as K579) to one of the two samples. The reason behind this additional control is that there are other DPP family members (such as Fibroblast Activating Protein [FAP] and intracellular DPP8 and 9) that might become exposed after tissue lysis, giving positive signals in the assay. K579 inhibits the activity of DPP4 at concentrations of $50 \mathrm{nM}$. Samples do not need to be incubated at length with K579, as inhibition with these concentrations occurs as soon as the reagents are mixed together. It is recommended to perform the assay using at least 4 biological replicates (e.g., biological samples from 4 mice) and 2 technical replicates per sample.

2. The representation of results can be done in relative fluorescent units (RFUs) by subtracting the RFU levels of the blank wells to all values. For representation of DPP4-specific activity a subtraction of the activity of samples incubated with K579 can be done. Alternatively, the results can be represented in Units of DPP activity. For this, please refer to the lot number of the recombinant DPP4 used for generation of the standard curve, as there should be stated the Units of activity per weight of enzyme. The values can then be extrapolated from the standard curve of the assay.

3. Detection of DPP4 activity in the entire body of the mouse can be done following intravenous injection of the Gly-Pro-aminoluciferin. This method can be used to study pharmacodynamics of DPP4 inhibition in vivo, following administration of DPP4 inhibitors, such as sitagliptin (Figure 1). Moreover, it is likely that this technique can be extrapolated to measure DPP4 activity in restricted tissues, such as inoculated luciferase-expressing tumors or transferred immune cells. It is recommended to perform the assay in at least 3 biological replicates.

\section{Recipes}

1. Prionex solution - Prepare fresh for each experiment 1x PBS supplemented with $0.1 \%$ Prionex

\section{Acknowledgments}

When using this protocol, please cite Barreira da Silva et al. (2015). Funding was provided by the Pasteur-Roux post-doctoral fellowship (RBdS), the Ligue Contre le Cancer and the Fondation ARC pour la recherche sur le cancer (MLA) and the French government's Invest in the Future Program, 
managed by the Agence Nationale de la Recherche (LabEx Immuno-Onco [RBdS, MAI MLA]). We thank M.A. Nicola (Plateforme d'imagerie dynamique, Institut Pasteur, Paris, France) for providing FVB CAG-luciferase transgenic mice. Animal experimental protocols were approved by the comité d'éthique pour l'expérimentation animale (The ethics committee for animal experimentation) Paris.

\section{References}

1. Barreira da Silva, R., Laird, M. E., Yatim, N., Fiette, L., Ingersoll, M. A. and Albert, M. L. (2015). Dipeptidylpeptidase 4 inhibition enhances lymphocyte trafficking, improving both naturally occurring tumor immunity and immunotherapy. Nat Immunol 16(8): 850-858. 